\title{
The effect of probiotic supplementation on gastrointestinal quality of life
}

\begin{abstract}
Purpose: "The purpose of this research study was to investigate the effect of Puritan's Pride probiotic on gastrointestinal quality of life of working adults aged 18 to 65years at the Loma Linda Support Services Building."

Methods: Working adults, 18 to 65 years of age, from the Loma Linda Support Services Building were eligible to participate in the study. Subjects were excluded if they were taking antibiotics or had taken antibiotics within the previous six months. Subjects who consented were given an initial Gastrointestinal Quality of Life Index (GIQLI) ${ }^{1}$ test and a probiotic supplement for 60days. Subjects' body composition was also tested during the initial visit. After the 60 days of supplementation, subjects returned to retake the GIQLI and to retest their body composition.
\end{abstract}

Results: Eleven subjects, 1 male and 10 female, reported for post intervention GIQLI and body composition testing. A mean increase of $10.7(\mathrm{SD}=11.1)$ in GIQLI scores was found after probiotic supplementation $(\mathrm{p}=.009)$. No significant differences were found in pre and post intervention measurements for BMI, body fat, and weight. The best predictor of post intervention GIQLI scores was pre intervention GIQLI scores.

Conclusion: There was an improvement in the related gastrointestinal quality of life in our subjects as shown through a significant increase in GIQLI scores and subjects' commentary. The best predictor of posttest GIQLI scores was pretest GIQLI scores.

Keywords: probiotic, supplement, supplementation, bacteria, micro biome, quality of life, gastrointestinal, GIQLI, gut, digestion

\author{
Volume 6 Issue 3 - 2017
}

Ernie Lin,' Jasmine K El Nabli,' Krysten A McCaughey,' Grenith J Zimmerman, ${ }^{2}$ Louise Schneider,' Jeje Noval'

'Department of Nutrition \& Dietetics, Loma Linda University, USA

${ }^{2}$ Department of Allied Health Studies, Loma Linda University, USA

Correspondence: Jeje Noval, Department of Nutrition \& Dietetics, Loma Linda University, USA, Email jnoval@llu.edu

Received: April 18, 2017| Published: May 04, 2017
Abbreviations: GERD, gastro esophageal reflux disease; IBD, inflammatory bowel disease; IBS, irritable bowel syndrome; LLUAHSC 101, loma linda support services building; GIQLI, gastro intestinal quality of life index; BMI, body mass index

\section{Introduction}

In the United States, immune-mediated and gut-related health issues are progressively increasing. ${ }^{2}$ According to the National Institute of Health, digestive diseases including, but not limited to, irritable bowel diseases and chronic constipation affect approximately 70million Americans annually. ${ }^{3}$ Because dysbiosis, or microbial imbalance inside the body, can lead to inflammatory and immune related disease development, achieving and maintaining optimal gut bacteria is advantageous in counteracting these inflammatory effects. ${ }^{2}$ Probiotic supplementation may aid in achieving the optimal balance of bacteria in the gut micro biome. These positive effects of probiotic supplementation have garnered the interest of many, as reflected in recent statistics provided by the National Center for Complementary and Integrative Health. "In 2012, the use of probiotics or prebiotics by adults in the United States was four times higher than in $2007 .{ }^{4}$

According to the Food and Agriculture Organization of the United Nations World Health Organization, probiotics are defined as "live microorganisms which when administered in adequate amounts confer a health benefit on the host. ${ }^{5}$ Microorganisms present in the gut make up the gut micro biome, and are involved in numerous functions necessary for physiological functioning, such as polysaccharide digestion, nutrient extraction, immune system development, protection against infections, synthesis of vitamins, angiogenesis regulation, and behavior development. ${ }^{6}$ This acquired micro biome is highly dynamic in nature and is affected by many factors, including but not limited to, diet, age, illness, medications, hormonal cycles, and lifestyle changes. ${ }^{6}$ The gut micro biome present in healthy adults has been shown to be more stable and diverse compared to that of infants and the elderly population, with Firmicutes and Bacteroidetes being the most abundant phyla of bacteria. ${ }^{6}$ A myriad of bacteria strains exist, but in order for a strain to be classified as a probiotic, it must be scientifically shown to have beneficial physiological effects, be safe for human consumption, derived from human origin, stable in both acid and bile, and adhere to intestinal mucosa. ${ }^{2}$ Lactobacillus $s p$. and Bifidobacterium $s p$. are the two most predominant strains studied in the literature because they have shown promising results in improving gastrointestinal quality of life.

Researchers have shown that probiotic use is positively correlated with gastrointestinal function regulation and gastrointestinal quality of life improvement. ${ }^{7}$ More specifically, probiotics "promote gut-barrier functions, give maturational signals for the gut-associated lymphoid tissues, and balance the generation of pro- and anti-inflammatory cytokines. ${ }^{2}$ Furthermore, researchers have found that probiotic supplementation decreases gastrointestinal transit time, increases stool frequency, and improves stool consistency with no adverse events. ${ }^{8}$ Lastly, in some patients with irritable bowel syndrome, probiotic supplementation has decreased symptoms in diarrhea-predominant irritable bowel syndrome, reduced bloating, and improved bowel movement consistency, all of which led to improved quality of life. ${ }^{9}$ 
In today's society, advancements in technology have led to more sedentary jobs and a demand for higher output in the workplace. This demand can often cause chronic stress in employees. Sedentary occupations and occupations performed indoors are associated with a higher risk of inflammatory bowel disease. This association may be caused by a lack of physical activity and the stress created by the demand for a higher output. ${ }^{10}$ Job stress has been shown to play a role in the development of gastric issues, contribute to negative health behaviors, and inhibit workers' abilities to make positive changes to lifestyle behaviors. ${ }^{11,12}$ Additionally, chronic stress associated with the workplace is a major risk factor in the pathogenesis of different diseases of the gastrointestinal tract including gastro esophageal reflux disease (GERD), peptic ulcer, functional dyspepsia, inflammatory bowel disease (IBD), and irritable bowel syndrome (IBS). ${ }^{1}$

Probiotics, available on the market, contain a variety of strains and differing dosages available on the market. Despite the number of probiotic choices, we chose to select and analyze one specific probiotic supplement brand in order to determine its effectiveness. Therefore, the purpose of this graduate student research study was to investigate the effect of Puritan's Pride probiotic on gastrointestinal quality of life in working adults aged 18 to 65 years.

\section{Materials and methods}

\section{Subjects}

Subjects were recruited through flyers posted within the Loma Linda Support Services Building (LLUAHSC 101), as well as through email. Authorized investigators were present on site to answer questions and check eligibility on specified dates. Subjects included a total of 11 employees from LLUAHSC 101 in the city of Loma Linda, California who had never taken a probiotic supplement in their lifetime and were between the ages of 18 to 65years. Subjects were excluded if they had taken antibiotics within the last six months. Only subjects from LLUAHSC 101 were included to limit variability in workplace behavior and activity level.

Potential subjects who responded to posters and emails were given an informed consent form during the initial meeting with employees and prior to the first administration of the Gastrointestinal Quality of Life Index (GIQLI) (Appendix A). The investigators were present to answer any questions. Subjects had 15 minutes to consider participating in the study. Subjects were chosen on a first come, first serve basis, as long as they fulfilled both inclusion and exclusion criteria. A total of 17 subjects were recruited, but only 11 subjects completed the study.

\section{Supplementation}

The probiotic supplement used was Puritan's Pride Probiotic 10. The capsules contained 20 billion live probiotic cultures from 10 probiotic strains. These 10 strains were Lactobacillus plantarum, Bifidobacterium bifidum, Lactobacillus rhamnosus, Lactobacillus bulgaricus, Lactobacillus salivarius, Lactobacillus brevis, Lactobacillus acidophilus, Bifidobacterium lactis, Lactobacillus paracasei, Lactobacillus casei. The remaining ingredients were gelatin, dicalcium phosphate, silica, vegetable magnesium stearate and some milk ingredients. Puritan's Pride manufactured the probiotic supplement used and the supplement was purchased online through their website (www.puritan.com).

\section{Gastrointestinal quality of life index}

Quality of life was measured using the Gastrointestinal Quality of Life Index (GIQLI) before and after a two-month intervention. The
GIQLI is a valid and well-known tool for assessing quality of life of patients with gastrointestinal complaints, and was developed by a team of four surgeons and three methodologists. ${ }^{13}$ The GIQLI consists of 36 questions which fall into the following five main categories: core symptoms, psychological, physical, social, and disease-specific. Core symptom questions include abdominal pain, epigastric fullness, bloating, flatus, belching, abdominal noises, bowel frequency, enjoyed or restricted eating, and fatigue. An example question is "How often during the past 2 weeks have you been troubled by uncontrollable stools?" Psychological questions encompass coping with stress, sadness, nervousness, happiness, and frustration. Physical items include feeling unwell or unfit trouble sleeping, changes in appearance, and loss in physical strength or endurance. Social questions inquire about completion of normal daily activities and recreational activities, as well as worsened personal relationships and impairment of sexual life. Lastly, disease-specific questions regarding frequency of issues regarding regurgitation, dysphagia, eating speed, nausea, diarrhea, bowel urgency, constipation, and blood in the stool are included. Each symptom is rated from 0 to 4 ( 0 being the worst and 4 being the best) with a maximum score of 144 points.(Table2)

Table I Summary of data from II subjects who completed the probiotic supplementation study

\begin{tabular}{|c|c|c|c|c|c|}
\hline & $\mathbf{N}$ & Minim & nMaximum & Mean & $\begin{array}{l}\text { Standard } \\
\text { deviation }\end{array}$ \\
\hline Age (Years) & 11 & 24 & 62 & 45.4 & 13 \\
\hline BMI & 11 & 23 & 38.3 & 29.78 & 5.13 \\
\hline Body Fat & 11 & 30.8 & 53 & $4 I .25$ & 8.07 \\
\hline Height & 11 & 60 & 71 & 65 & 3.5 \\
\hline Weight & 11 & 119.7 & 246.5 & 181.11 & | 44.58 \\
\hline Giqli Pretest & 11 & 71 & 136 & 102.4 & 19.4 \\
\hline Giqli Posttest & 11 & 83 & 140 & 113.1 & 20.8 \\
\hline $\begin{array}{l}\text { Change in Giqli } \\
\text { Scores }\end{array}$ & 11 & -5 & 32 & 10.7 & 11.1 \\
\hline $\begin{array}{l}\text { Percent Compliance } \\
\text { with Supplement }\end{array}$ & 10 & 75 & 100 & 92.8 & 9.4 \\
\hline
\end{tabular}

Table 2 Stepwise regression test for variables predicting GIQLI post test scores

\begin{tabular}{ll}
\hline Variable & P-Value* \\
\hline GIQLI pretest & 0.001 \\
Weight & 0.47 \\
BMI & 0.85 \\
Body Fat & 0.67 \\
\hline
\end{tabular}

*Stepwise regression test, significance at $p \leq .05$ 


\section{Procedures}

The experiment was a repeated-measures study in which 11 subjects were given a pretest prior to the intervention and a posttest following the intervention. Once consented, subjects were asked to fill out a questionnaire regarding current gastrointestinal quality of life. Subjects were then provided with a 60day supply (120 capsules) of Puritan's Pride probiotic supplement, consisting of a dosage of 40billion live cultures. Subjects were instructed to take two capsules daily in the morning until post testing.

\section{Intervention}

During the two months of the study, subjects:

a. Took two capsules of the Puritan's Pride probiotic supplement every morning

b. Received weekly email or phone reminders by subject's preference

Filled in a weekly diary regarding compliance with taking probiotic supplement and core symptoms while taking the probiotic supplement (Appendix B).

Continued supplementation on the following day for any days missed, and logged it in the diary.

Returned the diary at the final visit to the investigators.

Filled out the same GIQLI at the end of the two month duration.

\section{Data analysis}

All collected data was analyzed using SPSS ver. 24.0 (SPSS Inc., Chicago, IL, USA) and a p-value $\leq 0.05$ indicated statistical significance. Paired t-tests were used to determine significant changes between pre and post anthropometric measurements and GIQLI scores. Stepwise regression tests were used to find the best predictor of the post intervention GIQLI score.

\section{Results}

Eleven of the 17 subjects (64.7\%) completed the study. Six subjects were lost to follow up. The characteristics of the study group are summarized in Table 1. A female predominance (91\%) was noted among the 11 subjects, with only 1 male subject (9\%). The age of subjects ranged from 24 to 62 years, with a mean of 45.5 years. Based on the subjects' height and weight, a body mass index (BMI) status was able to be determined. The BMI of our subjects ranged from normal to obese, with the mean BMI being overweight. Overall, our typical subject was overweight, middle-aged, and 5' 5 " in height.

We examined the difference between pretest and posttest Gastrointestinal Quality of Life Index scores using a paired t-test. We found a mean increase in GIQLI scores of 10.7 (SD=11.1) which was significant $(\mathrm{p}=.009)$. Even with the one male subject removed, statistical analysis still confirmed a significant increase in GIQLI scores, with a mean increase of $9.4(\mathrm{SD}=10.7, \mathrm{p}=.02)$. Initially, we had planned on comparing the effect of probiotic supplementation for those with high initial GIQLI scores to those with low initial GIQLI scores; however, because of low participation and high variability, this comparison was not done.

We examined the differences between pre and post intervention measurements for BMI, body fat, and weight. The mean differences were as follows: change in BMI .10 kg/m2 ( $\mathrm{SD}=.34)$, change in body fat $-1.26 \%(\mathrm{SD}=4.49)$, change in weight. 63 pounds $(\mathrm{SD}=1.98)$. There was no significant difference in changes in BMI $(\mathrm{p}=.36)$, body fat $(\mathrm{p}=.36)$, or weight $(\mathrm{p}=.31)$.

From our stepwise regression tests, we found that the best predictor of posttest GIQLI scores was pretest GIQLI scores $(\mathrm{p}=.001)$. BMI $(\mathrm{p}=.85)$, body fat $(\mathrm{p}=.67)$, and weight $(\mathrm{p}=.47)$ were not significant predictors of post-test GIQLI scores.

\section{Discussion}

The findings from this study are consistent with current literature that probiotic supplementation is related to gastrointestinal function regulation and gastrointestinal quality of life improvement. ${ }^{7} \mathrm{We}$ observed a significant improvement in GIQLI scores after a twomonth intervention of two probiotic capsules daily. These results are consistent with a previous study's findings that probiotic supplementation decreases gastrointestinal transit time, increases stool frequency, and improves stool consistency with no adverse effects. $^{8}$

Although the media suggests that probiotics may promote weight loss, the present study did not find significant changes in BMI, body fat, or weight. This suggests that probiotic supplementation may not play a significant role in weight management in this population; however, although both occurred between the hours of 12:00PM and 2:00PM, anthropometric measurements of body fat mass may have been affected by subjects' hydration status per the instruction manual for the In Body scale.

The primary strength of our study is that our results add to the growing body of knowledge and research about probiotic supplementation. Our pilot study shows feasibility, cost effectiveness, and timeliness. Even with a small sample size, our findings revealed that probiotic supplementation can improve gastrointestinal quality of life. Future studies can use this evidence to justify the use of resources and support further investigation of probiotics on gastrointestinal quality of life. Another strength of our study is subject compliance when taking the probiotic supplements. Of those who successfully completed the study, compliance was high, mean of $92.8 \%$ compliant. Our study also supports literature findings that the use of probiotic supplementation seems to be safe and well tolerated by healthy adults. ${ }^{2}$

This study has a few limitations. First, due to the small sample size and dropout rate, findings may not have been fully representative of the population. Another limitation of this study is that the subjects may be affected by subject bias. In recent years, consuming pro biotics has gained popularity in the media as a weight loss method. Knowing this, subjects may have consciously or subconsciously followed healthier dietary habits while taking the probiotic supplement which may have affected the results.

In addition to the study's limitations, there are several confounding factors that may have influenced the results of this study. Two possible confounders for this study were physical exercise and daily caloric intake. These variables were not tracked or accounted for during the course of the study. The time of year during which this study took place could have also impacted results, as the study began in October and ended in mid-December. The Thanksgiving holiday and the holiday season in general could be confounding factors due to the commonly increased food intake during this time of year; however, no documentation of calorie intake was collected. 
We suggest a randomized controlled study design with a larger sample and the use of a placebo in order to eliminate subject bias and will give a more complete explanation of the potential benefits of probiotic supplementation and the mechanisms behind them. Future designs may also want to consider providing standardization for any qualitative data collected to account for subjects' perceived physiological improvements. Accounting for or standardizing physical activity and caloric intake may provide more representative results in relation the changes in anthropometric measures of BMI, body fat, and weight. Lastly, probiotic bioavailability must be considered, as different formulations and types of pro

biotics, proper storage, and supplement use with meals will greatly influence the viability of these supplements.

\section{Conclusion}

Our research study found significant differences between pretest and posttest GIQLI scores in employees from LLUAHSC 101. There was a significant improvement in the quality of life in our subjects after probiotic supplementation, with patients reporting improved regularity of bowel movements, and better sense of overall wellbeing. There were no significant differences in anthropometrics in our subjects after intervention. We found that the best predictor for our posttest GIQLI scores were pretest GIQLI scores. Overall, our research study adds to the body of knowledge and research surrounding probiotic supplementation for improved gastrointestinal quality of life.

\section{Acknowledgments} USA

Dr. Lee Berk, PhD, Allied Health Studies, Loma Linda University,

Dr. Gurinder Bains, PhD, Allied Health Studies, Loma Linda University, USA

\section{Conflict of interest}

Author declares that there is no conflict of interest.

\section{References}

1. Konturek P, Brzozowskif T, Konturek S. Stress and the gut: pathophysiology, clinical consequences, diagnostic approach and treatment options. J Physiol Pharmacol. 2011;62(6):591-599.

2. Isolauri E. Probiotics in human disease. Am J Clin Nutr. 2001;73(6):1142S-1146S.

3. Digestive Diseases Statistics for the United States. National Institute of Diabetes and Digestive and Kidney Diseases. 2016.

4. Use of Complementary Health Approaches in the U.S. National Institutes of Health. 2016.

5. Kechagia M, Basoulis D, Konstantopoulou S. Health benefits of probiotics: a review. ISRN Nutrition. 2013:1-7.

6. D'Argenio V, Salvatore F. The role of the gut micro biome in the healthy adult status. Clinica Chimica Acta. 2015;451:97-102.

7. Diop L, Guillou S, Durand H. Probiotic food supplement reduces stress-induced gastrointestinal symptoms in volunteers: a double-blind, placebo-controlled, randomized trial. Nutr Res. 2008;28(1):1-5.

8. Dimidi E, Christodoulides S, Fragkos K, et al. The effect of probiotics on functional constipation in adults: a systematic review and meta-analysis of randomized controlled trials. Am J Clin Nutr. 2014;100(4):1075-1084.

9. Hungin A, Mulligan C, Pot B, et al. Systematic review: probiotics in the management of lower gastrointestinal symptoms in clinical practice - an evidence-based international guide. Aliment Pharmacol Ther. 2013;38(8):864-886.

10. Sonnenberg A. Occupational distribution of inflammatory bowel disease among German employees. Gut. 1990;31(9):1037-1040.

11. Lihm H, Park S, Gong E, et al. Relationship between occupational stress and gastric disease in male workers. Korean $J$ Fam Med. 2012;33(5):311-319.

12. Nakao M. Work-related stress and psychosomatic medicine. Bio Psycho Social Medicine. 2010;4(1):4.

13. Eypasch E, Williams J, Wood-Dauphinee S. Gastrointestinal quality of life index: development, validation and application of a new instrument. British Journal of Surgery. 1995;82(2):216-222. 